\title{
The Warp Computer: Architecture, Implementation, and Performance
}

\author{
Marco Annaratone, Emmanuel Arnould, Thomas Gross, \\ H. T. Kung, Monica Lam, Onat Menzilcioglu, Jon A. Webb
}

CMU-RI-TR-87-18

\author{
Department of Computer Science \\ The Robotics Institute \\ Carnegie Mellon University \\ Pittsburgh, Pennsylvania 15213
}

July 1987

Copyright $\odot 1987$ Carnegie Mellon University

The research was supported in part by Defense Advanced Research Projects Agency (DoD) monitored by the Space and Naval Warfare Systems Command under Contract N00039-85-C-0134, and in part by the Office of Naval Research under Contracts N00014-87-K-0385 and N00014-87-K-0533.

Warp is a service mark of Camegie Mellon University. UNIX is a trademark of AT\&T Bell Laboratories. Sun-3 is a tradernark of Sun Microsystems.

To appear in IEEE Transactions on Computers for a special issue on supercomputers. 


\section{Table of Contents}

1 Introduction

2 Warp system overview

3 Warp array architecture

4 Warp cell architecture

4.1 Inter-cell communication

4.1.1 Programmable delay

4.1.2 Flow control

4.1.3 Input control

4.1.4 Randomly accessible queues

4.1.5 Queue size

4.2 Control path

4.3 Data path

4.3.1 Floating-point units

4.3.2 Crossbar

4.3.3 Data storage blocks

4.3.4 Address generation

5 Warp cell and IU implementation

6 Host system

6.1 Host I/O bandwidth

6.2 Host software

7 Programming Warp

7.1 The $W 2$ language

7.2 Problem partitioning

7.2.1 Input partitioning

7.2.2 Output partitioning

7.2.3 Pipelining

8 Evaluation

8.1 Performance data

8.2 Architectural Alternatives

8.2.1 Programming model

8.2.2 Processor I/O bandwidth and topology

8.2.2 Processor $1 / O$ bandwidth and topology 


\section{List of Figures}

Figure 1: Warp system overview

Figure 2: Warp cell data path

Figure 3: Compile-time flow control

Figure 4: Merging equal-length loops with an offset: (a) original loops, (b) execution trace, and (c) merged loop.

Figure 5: Merging loops with different lengths: (a) original loops, (b) execution trace, 8 and (c) merged loop.

Figure 6: Host of the Warp machine

Figure 7: Example program

12

Figure 8: Performance distribution of a set of $72 \mathrm{~W} 2$ programs 


\section{List of Tables}

Table 1: Implementation metrics for Warp cell

Table 2: Implementation metrics for IU

Table 3: Measured speedups on the wire-wrapped prototype Warp machine

Table 4: Performance of specific algorithms on the wire-wrapped prototype Warp machine 


\begin{abstract}
The Warp machine is a systolic array computer of linearly connected cells, each of which is a programmable processor capable of performing 10 million floating-point operations per second (10 MFLOPS). A typical Warp array includes 10 cells, thus having a peak computation rate of 100 MFLOPS. The Warp array can be extended to include more cells to accommodate applications capable of using the increased computational bandwidth. Warp is integrated as an attached processor into a UNIX host system. Programs for Warp are written in a high-level language supported by an optimizing compiler.

The first 10-cell prototype was completed in February 1986; delivery of production machines started in April 1987. Extensive experimentation with both the prototype and production machines has demonstrated that the Warp architecture is effective in the application domain of robot navigation, as well as in other fields such as signal processing, scientific computation, and computer vision research. For these applications, Warp is typically several hundred times faster than a VAX 11/780 class computer.

This paper describes the architecture, implementation and performance of the Warp machine. Each major architectural decision is discussed and evaluated with system, software and application considerations. The programming model and tools developed for the machine are also described. The paper concludes with performance data for a large number of applications.
\end{abstract}




\section{Introduction}

The Warp machine is a high-performance systolic array computer designed for computation-intensive applications. In a typical configuration, Warp consists of a linear systolic array of 10 identical cells, each of which is a 10 MFLOPS programmable processor. Thus a system in this configuration has a peak performance of 100 MFLOPS.

The Warp machine is an attached processor to a general purpose host running the UNIX operating system. Warp can be accessed by a procedure call on the host, or through an interactive, programmable command interpreter called the Warp shell [8]. A high-level language called W2 is used to program Warp; the language is supported by an optimizing compiler $[12,23]$.

The Warp project started in 1984. A 2-cell system was completed in June 1985 at Carnegie Mellon. Construction of two identical 10-cell prototype machines was contracted to two industrial partners, GE and Honeywell. These prototypes were built from off-the-shelf parts on wire-wrapped boards. The first prototype machine was delivered by GE in February 1986, and the Honeywell machine arrived at Carnegie Mellon in June 1986. For a period of about a year during 1986-87, these two prototype machines were used on a daily basis at Carnegie Mellon.

We have implemented application programs in many areas, including low-level vision for robot vehicle navigation, image and signal processing, scientific computing, magnetic resonance imagery (MRI) image processing, radar and sonar simulation, and graph algorithms $[3,4]$. In addition, we have developed a significant low-level image library of about one hundred routines [17]. Our experience has shown that Warp is effective in these applications; Warp is typically several hundred times faster than a VAX 11/780 class computer.

Encouraged by the performance of the prototype machines, we have revised the Warp architecture for reimplementation on printed circuit (PC) boards to allow faster and more efficient production. The revision also incorporated several architectural improvements. The production Warp machine is referred as the PC Warp in this paper. The PC Warp is manufactured by GE, and is available at about $\$ 350,000$ per machine. The first PC Warp machine was delivered by GE in April 1987 to Carnegie Mellon.

This paper describes the architecture of the Warp machine, the rationale of the design and the implementation, and performance measurements for a variety of applications. The organization of the paper is as follows. We first present an overview of the system. We then describe how the overall organization of the array allows us to use the cells efficiently. Next we focus on the cell architecture: we discuss each feature in detail, explaining the design and the evolution of the feature. We conclude this section on the cell with a discussion of hardware implementation issues and metrics. We then describe the architecture of the host system. To give the reader some idea of how the machine can be programmed, we describe the W2 programming language, and some general methods of partitioning a problem onto a processor array that have worked well for Warp. To evaluate the Warp machine architecture, we present performance data for a variety of applications on Warp, and a comparison of the architecture of Warp with other parallel machines.

\section{Warp system overview}

There are three major components in the system - the Warp processor array (Warp array), the interface unit (IU), and the host, as depicted in Figure 1. The Warp array performs the computation-intensive routines such as low-level vision routines or matrix operations. The IU handles the input/output between the array and the host, and can generate addresses (Adr) and control signals for the Warp array. The host supplies data to and receives results from the array. In addition, it executes those parts of the application programs which are not mapped onto the Warp array. For example, the host may perform decision-making processes in robot navigation or evaluate convergence criteria in iterative methods for solving systems of linear equations. 


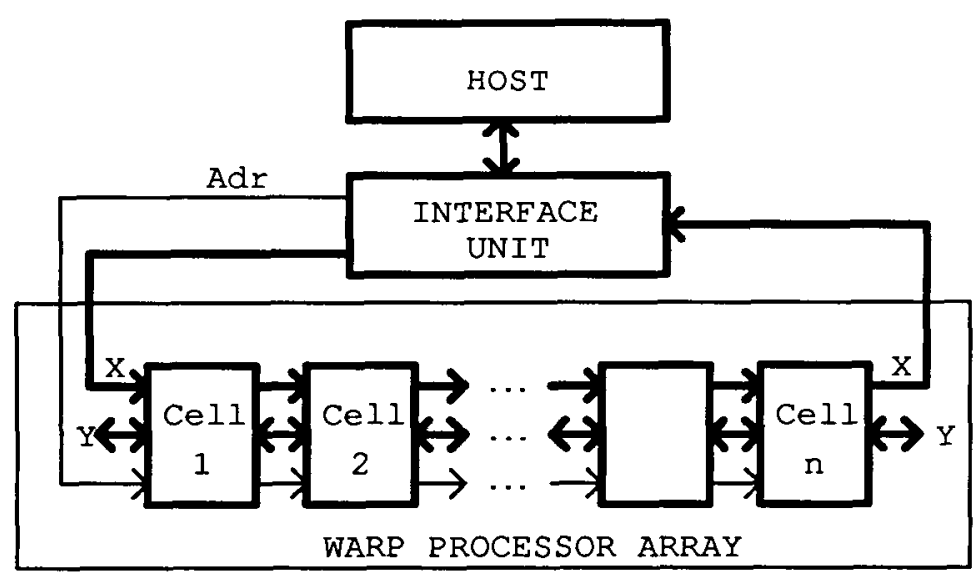

Figure 1: Warp system overview

The Warp array is a linear systolic array with identical cells called Warp cells, as shown in Figure 1. Data flow through the array on two communication channels ( $\mathrm{X}$ and $\mathrm{Y}$ ). Those addresses for cells' local memories and control signals that are generated by the IU propagate down the Adr channel. The direction of the $Y$ channel is statically configurable. This feature is used, for example, in algorithms that require accumulated results in the last cell to be sent back to the other cells (e.g., in back-solvers), or require local exchange of data between adjacent cells (e.g., in some implementations of numerical relaxation methods).

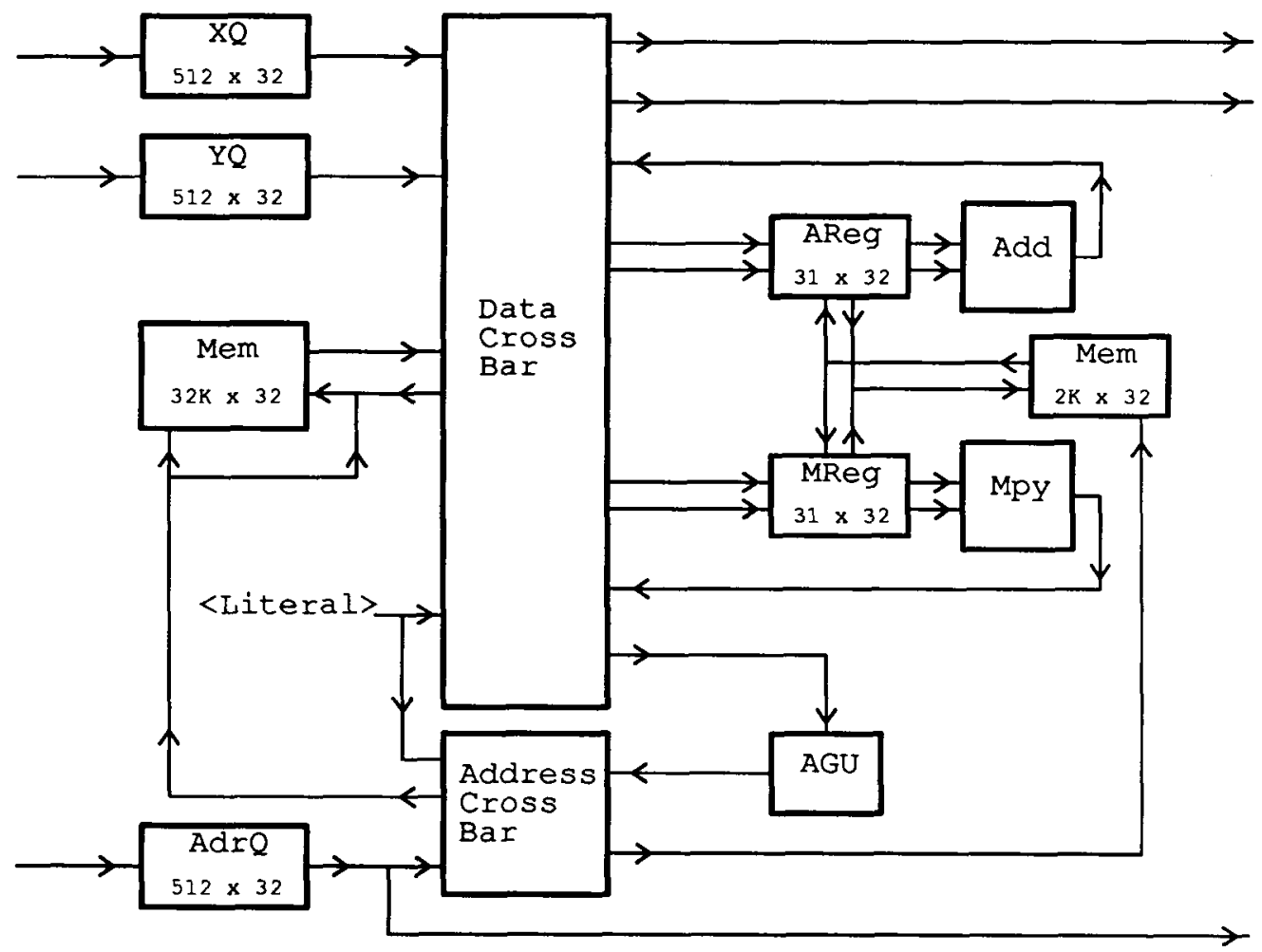

Figure 2: Warp cell data path

Each Warp cell is implemented as a programmable horizontal micro-engine, with its own microsequencer and program memory for $8 \mathrm{~K}$ instructions. The Warp cell data path, as depicted in Figure 2, consists of a 32-bit floating-point multiplier (Mpy) and a 32-bit floating-point adder (Add), two local memory banks for resident and 
temporary data (Mem), a queue for each inter-cell communication channel (XQ, YQ, and AdrQ), and a register file to buffer data for each floating-point unit (AReg and MReg). All these components are connected through a crossbar. Addresses for memory access can be computed locally by the address generation unit (AGU), or taken from the address queue (AdrQ).

The Warp cell datapath is similar to the datapath of the Floating Point Systems AP-120B/FPS-164 line of processors [9], which are also used as attached processors. Both the Warp cell and any of these FPS processors contain two floating-point units, memory and an address generator, and are oriented towards scientific computing and signal processing. In both cases, wide instruction words are used for a direct encoding of the hardware resources, and software is used to manage the parallelism (that is, to detect parallelism in the application code, to use the multiple functional units, and to pipeline instructions). The Warp cell differs from these earlier processors in two key aspects: the full crossbar of the Warp cell provides a higher intra-cell bandwidth, and the $\mathrm{X}$ and $\mathrm{Y}$ channels with their associated queues provide a high inter-cell bandwidth, which is unique to the Warp array architecture.

The host consists of a Sun-3 workstation that serves as the master controller of the Warp machine, and a VME-based multi-processor external host, so named because it is external to the workstation. The workstation provides a UNIX environment for running application programs. The external host controls the peripherals and contains a large amount of memory for storing data to be processed by the Warp array. It also transfers data to and from the Warp array and performs operations on the data when necessary, with low operating system overhead.

Both the Warp cell and IU use off-the-shelf, TTL-compatible parts, and are each implemented on a $15^{\prime \prime} \times 17^{\prime \prime}$ board. The entire Warp machine, with the exception of the Sun-3, is housed in a single 19" rack, which also contains power supplies and cooling fans. The machine typically consumes about $1,800 \mathrm{~W}$.

\section{Warp array architecture}

In the Warp machine, parallelism exists at both the array and cell levels. This section discusses how the Warp architecture is designed to allow efficient use of the array level parallelism. Architectural features to support the cell level parallelism are described in the next section.

The key features in the architecture that support the array level parallelism are: simple topology of a linear array, powerful cells with local program control, large data memory for each cell, and high inter-cell communication bandwidth. These features support several problem partitioning methods important to many applications [21, 22]. More details on the partitioning methods are given in Section 7.2, and a sample of applications using these methods are listed in Section 8.

A linear array is easier for a programmer to use than higher dimensional arrays. Many algorithms in scientific computing and signal processing have been developed for linear arrays [18]. Our experience of using Warp for low-level vision has also shown that a linear organization is suitable in the vision domain as well. A linear array is easy to implement in hardware, and demands a low external $\mathrm{I} / \mathrm{O}$ bandwidth since only the two end-cells communicate with the outside world. Moreover, a linear array consisting of powerful, programmable processors with large local memories can efficiently simulate other interconnection topologies. For example, a single Warp cell can be time multiplexed to perform the function of a column of cells, so that the linear Warp array can implement a two-dimensional systolic array.

The Warp array can be used for both fine-grain and large-grain parallelism. It is efficient for fine-grain parallelism needed for systolic processing, because of its high inter-cell bandwidth. The I/O bandwidth of each cell is higher than that of other processors with similar computational power. Each cell can transfer 20 million 32-bit words ( $80 \mathrm{Mbytes})$ per second to and from its neighboring cells, in addition to 20 million 16-bit addresses. This 
high inter-cell communication bandwidth permits efficient transfers of large volumes of intermediate data between neighboring cells.

The Warp array is efficient for large-grain parallelism because it is composed of powerful cells. Each cell is capable of operating independently; it has its own program sequencer and program memory of $8 \mathrm{~K}$ instructions. Moreover, each cell has $32 \mathrm{~K}$ words of local data memory, which is large for systolic array designs. For a given $\mathrm{I} / \mathrm{O}$ bandwidth, a larger data memory can sustain a higher computation bandwidth for some algorithms [20].

Systolic arrays are known to be effective for local operations, in which each output depends only on a small corresponding area of the input. The Warp array's large memory size and its high inter-cell I/O bandwidth enable it to perform global operations in which each output depends on any or a large portion of the input [21]. The ability of performing global operations as well significantly broadens the applicability of the machine. Examples of global operations are fast Fourier transform (FFT), image component labeling, Hough transform, image warping, and matrix computations such as LU decomposition or singular value decomposition (SVD).

Because each Warp cell has its own sequencer and program memory, the cells in the array can execute different programs at the same time. We call computation where all cells execute the same program homogeneous, and heterogeneous otherwise. Heterogeneous computing is useful for some applications. For example, end-cells may operate differently from other cells to deal with boundary conditions. Or, in a multi-function pipeline [13], different sections of the array perform different functions, with the output of one section feeding into the next as input.

\section{Warp cell architecture}

This section describes the design and the evolution of the architectural features of the cell. Some of these features were significantly revised when we re-implemented Warp in PC boards. For the wire-wrapped prototype, we omitted some architectural features that are difficult to implement and are not necessary for a substantial fraction of application programs [1]. This simplification in the design permitted us to gain useful experience in a relatively short time. With the experience of constructing and using the prototype, we were able to improve the architecture and expand the application domain of the production machine.

\subsection{Inter-cell communication}

Each cell communicates with its left and right neighbors through point-to-point links, two for data and one for addresses. A queue with a depth of 512 words is associated with each link (XQ, YQ and AdrQ in Figure 2) and is placed in the data path of the input cell. The size of the queue is just large enough to buffer one or two scan-lines of an image, which is typically of size $512 \times 512$ or $256 \times 256$. The ability to buffer a complete scan-line is important for the efficient implementation of some algorithms such as two-dimensional convolutions [19]. Words in the queues are 34 bits wide; along with each 32-bit data word, the sender transmits two bits of control signal that can be tested by the receiver.

Flow control for the communication channels is implemented in hardware. When a cell tries to read from an empty queue, it is blocked until a data item arrives. Similarly, when a cell tries to write to a full queue of a neighboring cell, the writing cell is blocked until some data is removed from the full queue. The blocking of a cell is transparent to the program; the state of all the computational units on the data path freezes for the duration the cell is blocked. Only the cell that tries to read from an empty queue or to deposit a data item into a full queue is blocked. All other cells in the array continue to operate normally. The data queues of a blocked cell are still able to accept input; otherwise a cell blocked on an empty queue will never become unblocked.

The implementation of run-time flow control by hardware has two implications: First, we need two clock 
generators - one for the computational units whose states freeze when a cell is blocked, and one for the queues. Second, since a cell can receive data from either of its two neighbors, it can block as a result of the status of the queues in either neighbor, as well as its own. This dependence on other cells adds serious timing constraints to the design since clock control signals have to cross board boundaries. The complexity will be further discussed in Section 5.

The inter-cell communication mechanism is the most revised feature on the cell; it has evolved from primitive programmable delay elements to queues without any flow control hardware, and finally to the run-time flowcontrolled queues. In the following, we step through the different design changes.

\subsubsection{Programmable delay}

In an early design, the input buffer on each communication channel of a cell was a programmable delay element. In a programmable delay element, data are latched in every cycle and they emerge at the output port a constant number of cycles later. This structure is found in many systolic algorithm designs to synchronize or delay one data stream with respect to another. However, programmable high-performance processors like the Warp cells require a more flexible buffering mechanism. Warp programs do not usually produce one data item every cycle; a clocking discipline that reads and writes one item per cycle would be too restrictive. Furthermore, a constant delay through the buffer means that the timing of data generation must match exactly that of data consumption. Therefore, the programmable delays were replaced by queues to remove the tight coupling between the communicating cells.

\subsubsection{Flow control}

Queues allow the receiver and sender to run at their own speeds provided that the receiver does not read past the end of the queue and the sender does not overflow the queues. There are two different flow control disciplines, run-time and compile-time flow-control. As discussed above, hardware support for run-time flow control can be difficult to design, implement and debug. Alternatively, for a substantial set of problems in our application domain, compile-time flow control can be implemented by generating code that requires no run-time support. Therefore, we elected not to support run-time flow control in the prototype. This decision permitted us to accelerate the implementation and experimentation cycle. Run-time flow control is provided in the production machine, so as to widen the application domain of the machine.

Compile-time flow control can be provided for all programs where data only flow in one direction through the array and where the control flow of the programs is not data dependent. Data dependent control flow and two-way data flow can also be allowed for programs satisfying some restrictions [6]. Compile-time flow control is implemented by skewing the computation of the cells so that no receiving cell reads from a queue before the corresponding sending cell writes to it. For example, suppose two adjacent cells each execute the following program:

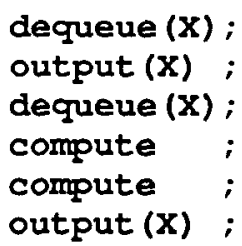

In this program, the first cell removes a data item from the $\mathbf{X}$ queue (dequeue $(\mathrm{X})$ ) and sends it to the second cell on $\mathbf{x}$ (output $(x)$ ). The first cell then removes a second item, and forwards the result to the second cell after 2 cycles of computation. For this program, the second cell needs to be delayed by 3 cycles to ensure that the dequeue of the second cell never overtakes the corresponding output of the first cell, and the compiler will insert the necessary nops, as shown in Figure 3. 
First cell
dequeue $(\mathrm{X})$;
output $(\mathrm{X})$;
dequeue $(\mathrm{X})$;
compute ;
compute ;
output $(\mathrm{X})$;
Second cell

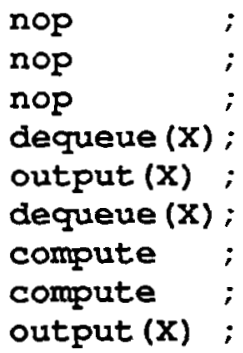

Figure 3: Compile-time flow control

Run-time flow control expands the application domain of the machine and often allows the compiler to produce more efficient code; therefore it is provided in the production machine. Without run-time flow control, wHILE loops and FOR loops with computed loop bounds on the cells cannot be implemented. That is, only loops with compiletime constant bounds can be supported. This restriction limits the class of programs executable on the machine. Moreover, many programs for the prototype machines can be made more efficient and easier to write on the production machine by replacing the FOR loops with WHLE loops. Instead of executing a fixed number of iterations to guarantee convergence, the iteration can be stopped as soon as the termination condition is met. The compiler can produce more efficient code since compile-time flow control by skewing delays the receiving cell sufficiently to guarantee correct behavior, but this delay is not necessarily the minimum delay needed. Run-time flow control will dynamically find the minimum bound.

\subsubsection{Input control}

In the current design, latching of data into a cell's queue is controlled by the sender, rather than by the receiver. As a cell sends data to its neighbor, it also signals the receiving cell's input queue to accept the data.

In our first 2-cell prototype machine, input data were latched under the microinstruction control of the receiving cell. This implied that inter-cell communication required close cooperation between the sender and the receiver; the sender presented its data on the communication channel, and in the same clock cycle the receiver latched in the input. This design was obviously not adequate if flow control was supported at run time; in fact, we discovered that it was not adequate even if flow control was provided at compile time. The tight coupling between the sender and the receiver greatly increased the code size of the programs. The problem was corrected in subsequent implementations by adopting the design we currently have, that is, the sender provides the signal to the receiver's queue to latch in the input data.

In the above discussion of the example of Figure 3, it was assumed that the control for the second cell to latch in input was sent with the output data by the first cell. If the second cell were to provide the input control signals, we would need to add an input operation in its microprogram for every output operation of the first cell, at exactly the cycle the operation takes place. Doing so, we obtain the following program for the second cell:

$\begin{array}{ll}\text { input (x) } & \text { nop ; } \\ & \text { nop ; } \\ & \text { dequeue (x); } \\ \text { output (x) ; } & \text { dequeue (x); } \\ \text { compute ; } & \text { compute ; } \\ \text { output (x) ; }\end{array}$


Each line in the program is a micro-instruction; the first column contains the Input operations to match the Output operations of the first cell, and the second column contains the original program.

Since the input sequence follows the control flow of the sender, each cell is logically executing two processes: the input process, and the original computation process of its own. These two processes must be merged into one since there is only one sequencer on each cell. If the programs on communicating cells are different, the input process and the cell's own computation process are different. Even if the cell programs are identical, the cell's computation process may need to be delayed with respect to the input process because of compile-time flow control as described above. As a result, we may need to merge control constructs from different parts of the program. Merging two equal-length loops, with an offset between their initiation times, requires loop unrolling and can result in a three-fold increase in code length. Figure 4 illustrates this increase in code length when merging two identical loops of $n$ iterations. Numbers represent operations of the input process, and letters represent the computation process. If two iterative statements of different lengths are overlapped, then the resulting code size can be of the order of the least common multiple of their lengths. For example, in Figure 5, a 2-instruction loop of $3 n$ iterations is merged with a 3 -instruction loop of $2 n$ iterations. Since 6 is the minimum number of cycles before the combined sequence of operations repeats itself, the resulting merged program is a 6-instruction loop of $n$ iterations.

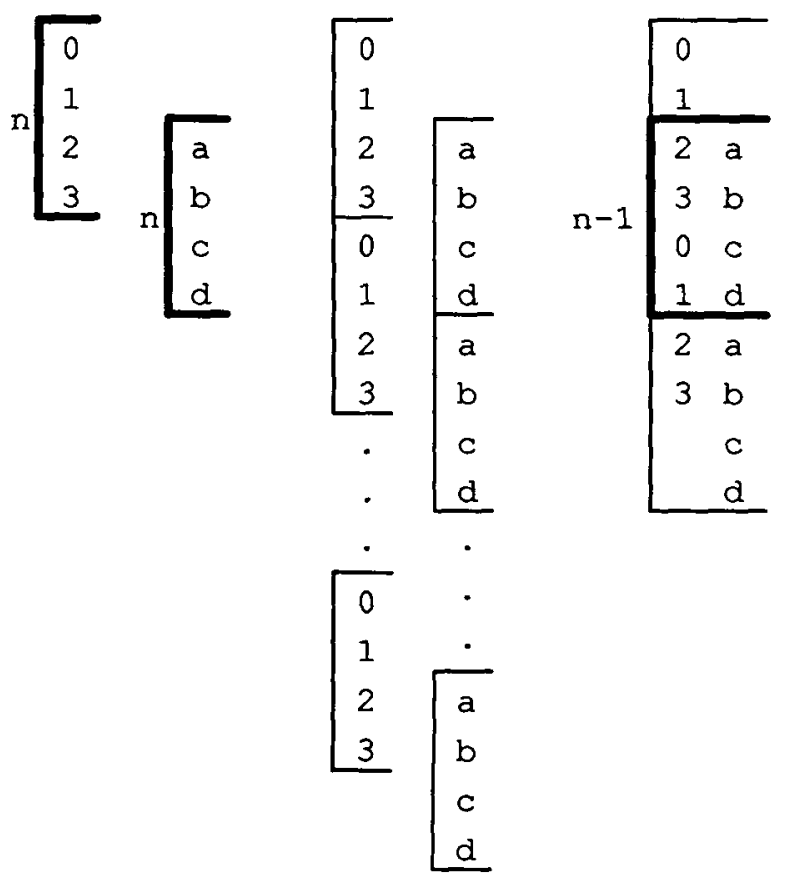

(a)

(b)

(c)

Figure 4: Merging equal-length loops with an offset:

(a) original loops, (b) execution trace, and (c) merged loop.

\subsubsection{Randomly accessible queues}

The queues in all the prototype machines are implemented with RAM chips, with hardware queue pointers. Furthermore, there was a feedback path from the data crossbar back to the queues, because we intended to use the queues as local storage elements as well [1]. Since the pointers must be changed when the queue is accessed randomly, and there is only a single pair of queue pointers, it is impossible to multiplex the use of the buffer as a communication queue and its use as a local storage element. Therefore, the queues in the production machine are now implemented by a FIFO chip. This implementation allows us to increase the queue size from 128 to 512 words, with board space left over for other improvements as well. 


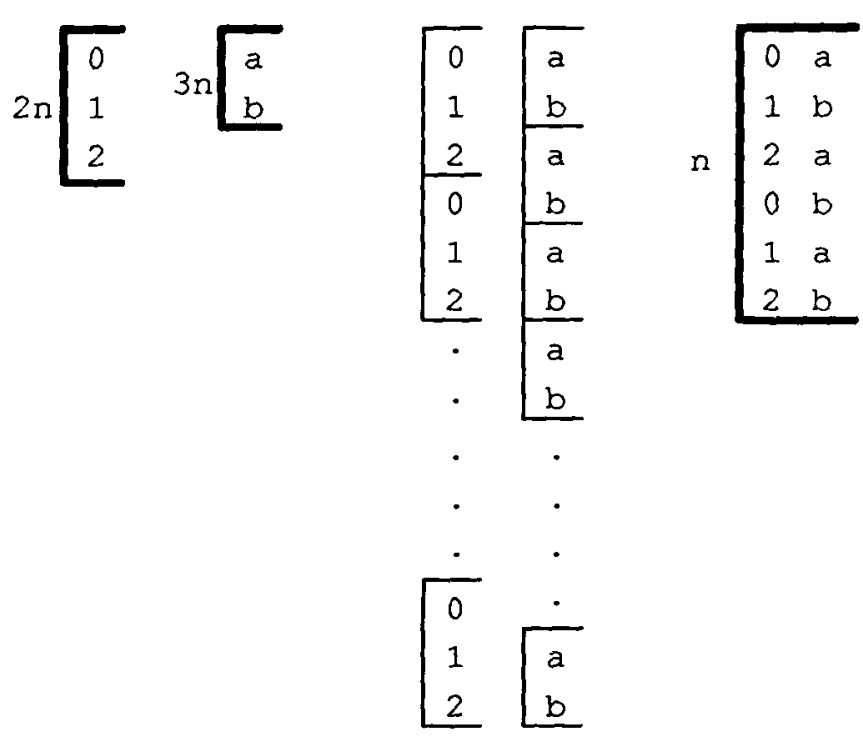

(a)

(b)

(c)

Figure 5: Merging loops with different lengths:

(a) original loops, (b) execution trace, and (c) merged loop.

\subsubsection{Queue size}

The size of the queues is an important factor in the efficiency of the array. Queues buffer the input for a cell and relax the coupling of execution in communicating cells. Although the average communication rate between two communicating cells must balance, a larger buffer allows the cells to receive and send data in bursts at different times.

The long queues allow the compiler to adopt a simple code optimization strategy [23]. The throughput for a unidirectional array is maximized by simply optimizing the individual cell programs provided that sufficient buffering is available between each pair of adjacent cells. In addition, some algorithms, such as two-dimensional convolution mentioned above, require large buffers between cells. If the queues are not large enough, a program must explicitly implement buffers in local memory.

\subsection{Control path}

Each Warp cell has its own local program memory and sequencer. This is a good architectural design even if the cells all execute the same program, as in the case of the prototype Warp machine. The reason is that it is difficult to broadcast the microinstruction words to all the cells, or to propagate them from cell to cell, since the instructions contain a large number of bits. Moreover, even if the cells execute the same program, the computations of the cells are often skewed so that each cell is delayed with respect to its neighboring cell. This skewed computation model is easily implemented with local program control. The local sequencer also supports conditional branching efficiently. In SIMD machines, branching is achieved by masking. The execution time is equivalent to the sum of the execution time of the then-clause and the else-clause of a branch. With local program control, different cells may follow different branches of a conditional statement depending on their individual data; the execution time is the execution time of the clause taken.

The Warp cell is horizontally microcoded. Each component in the data path is controlled by a dedicated field; this orthogonal organization of the microinstruction word makes scheduling easier since there is no interference in the schedule of different components. 


\subsection{Data path}

\subsubsection{Floating-point units}

Each Warp cell has two floating-point units, one multiplier and one adder, implemented with commercially available floating-point chips [35]. These floating-point chips depend on extensive pipelining to achieve high performance. Both the adder and multiplier have 5-stage pipelines. General purpose computation is difficult to implement efficiently on deeply pipelined machines, because data-dependent branching is common. There are less data dependency in numerical or computer vision programs, and we developed scheduling techniques that use the pipelining efficiently. Performance results are reported in Section 8.

\subsubsection{Crossbar}

Experience with the Programmable Systolic Chip showed that the internal data bandwidth is often the bottleneck of a systolic cell [11]. In the Warp cell, the two floating-point units can consume up to four data items and generate two results per cycle. Several data storage blocks interconnected with a crossbar support this high data processing rate. There are six input and eight output ports connected to the crossbar switch; up to six data items can be transferred in a single cycle, and an output port can receive any data item. The use of the crossbar also makes compilation easier when compared to a bus-based system since conflicts on the use of one or more shared busses can complicate scheduling tremendously.

Custom chip designs that combine the functionality of the crossbar interconnection and data buffers have been proposed [16,28]. In the interconnection chip designed for polycyclic architectures [28], a "queue" is associated with each cross point of the crossbar. In these storage blocks, data are always written at the end of the queue; however, data can be read, or removed, from any location. The queues are compacted automatically whenever data is removed. The main advantage of this design is that an optimal code schedule can be readily derived for a class of inner loops [27]. In the Warp cell architecture, we chose to use a conventional crossbar with data buffers only for its outputs (the AReg and MReg register files in Figure 2), because of the lower hardware cost. Near-optimal schedules can be found cheaply using heuristics [23].

\subsubsection{Data storage blocks}

As depicted by Figure 2, the local memory hierarchy includes a local data memory, a register file for the integer unit (AGU), two register files (one for each floating-point unit), and a backup data memory. Addresses for both data memories come from the address crossbar. The local data memory can store $32 \mathrm{~K}$ words, and can be both read and written every ( $200 \mathrm{~ns}$ ) cycle. The capacity of the register file in the AGU unit is 64 words. The register files for the floating-point units each hold 31 usable words of data. (The register file is written to in every cycle, so that one word is used as a sink for those cycles without useful write operations). They are 5-ported data buffers and can each accept two data items from the crossbar and deliver two operands to the functional units every cycle. The additional ports are used for connecting the register files to the backup memory. This backup memory contains $2 \mathrm{~K}$ words and is used to hold all scalars, floating-point constants, and small arrays. The addition of the backup memory increases memory bandwidth and improves throughput for those programs operating mainly on local data.

\subsubsection{Address generation}

As shown in Figure 2, each cell contains an integer unit (AGU) that is used predominantly as a local address generation unit. The AGU is a self-contained integer ALU with 64 registers. It can compute up to two addresses per cycle (one read address and one write address).

The local address generator on the cell is one of the enhancements that distinguish the PC Warp machine from the prototype. In the prototype, data independent addresses are generated on the $\Pi U$ and propagated down the cells. 
Data dependent addresses were computed locally on each cell using the floating-point units. The IU of the prototype had the additional task of generating the loop termination signals for the cells. These signals are propagated along the Adr channel to the cells in the Warp array.

There was not enough space on the wire-wrapped board to include local address generation capability on each Warp cell. Including an AGU requires board space not only for the AGU itself, but also for the bits in the instruction word for controlling it. An AGU was area expensive at the time the prototype was designed, due to the lack of VLSI parts for the AGU functions. The address generation unit in the prototype IU uses AMD2901 parts which contain 16 registers. Since this number of registers is too small to generate complicated addressing patterns quickly, the ALU is backed up by a table that holds up to $16 \mathrm{~K}$ pre-computed addresses. This table is too large to replicate on all the cells. The address generation unit on the PC Warp cells is a new VLSI component (IDT-49C402), which combines the register file and ALU on a single chip. The large number of registers makes the back-up table unnecessary for most addressing patterns, so that the AGU is much smaller and can be replicated on each cell of the production machine.

The prototype was designed for applications where all cells execute the same program with data independent loop bounds. However, not all such programs could be supported due to the size of the address queue. In the pipelining mode, where the cells implement different stages of a computation pipeline, a cell does not start executing until the preceding cell is finished with the first set of input data. The size of the address queue must at least equal the number of addresses and control signals used in the computation of the data set. Therefore the size of the address queues limits the number of addresses buffered, and thus the grain size of parallelism.

For the production machine, each cell contains an AGU and can generate addresses and loop control signals efficiently. This improvement allows the compiler to support a much larger class of applications. We have preserved the address generator and address bank on the IU (and the associated Adr channel, as shown in Figure 1). Therefore, the IU can still support those homogeneous computations that demand a small set of complicated addressing patterns that can be conveniently stored in the address bank.

\section{Warp cell and IU implementation}

The Warp array architecture operates on 32-bit data. All data channels in the Warp array, including the internal data path of the cell, are implemented as 16-bit wide channels operating at 100 ns. There are two reasons for choosing a 16-bit time-multiplexed implementation. First, a 32-bit wide hardware path would not allow implementing one cell per board. Second, the $200 \mathrm{~ns}$ cycle time dictated by the Weitek floating-point chips (at the time of design) allows the rest of the data path to be time multiplexed. This would not have been possible if the cycle time of the floating-point chips were under $160 \mathrm{~ns}$. The micro-engine operates at $100 \mathrm{~ns}$ and supports high and low cycle operations of the data path separately.

All cells in the array are driven from a global $20 \mathrm{MHz}$ clock generated by the IU. To allow each cell to block individually, a cell must have control over the use of the global clock signals. Each cell monitors two concurrent processes: the input data flow (I process) and the output data flow (O process). If the input data queue is empty, the I process flow must be suspended before the next read from the queue. Symmetrically, the $O$ process is stopped before the next write whenever the input queue of the neighboring cell is full. Stopping the $I$ or $O$ process pauses all computation and output activity, but the cell continues to accept input. There is only a small amount of time available between detection of the queue full/empty status and blocking the read/write operation. Since the cycle time is only $100 \mathrm{~ns}$, this tight timing led to race conditions in an early design. This problem has been solved by duplicating on each cell the status of the I/O processes of the neighboring cells. In this way, a cell can anticipate a queue full/empty condition and react within a clock cycle. 
A large portion of the internal cell hardware can be monitored and tested using built-in serial diagnostic chains under control of the $\mathrm{IU}$. The serial chains are also used to download the Warp cell programs. Identical programs can be downloaded to all cells at a rate of $95 \mu \mathrm{s}$ per instruction from the workstation and about $66 \mu \mathrm{s}$ per instruction from the external host. Starting up a program takes about $9 \mathrm{~ms}$.

The Warp cell consists of six main blocks: input queues, crossbar, processing elements, data memory, address generator, and micro-engine. Table 1 presents the contribution of these blocks to the implementation of the Warp cell. The micro-engine includes the program memory ( $8 \mathrm{~K}$ instruction words of $272 \mathrm{bits}$, including parity). The Warp cell consumes $94 \mathrm{~W}$ (typical) and $136 \mathrm{~W}$ (maximum).

\begin{tabular}{|l|c|c|}
\hline \multicolumn{1}{|c|}{ Block in Warp cell } & Chip count & Area contribution (\%) \\
\hline Queues & 22 & 9 \\
Crossbar & 32 & 11 \\
Processing elements and registers & 12 & 10 \\
Data memory & 31 & 9 \\
Local address generator & 13 & 6 \\
Micro-engine & 90 & 35 \\
Other & 55 & 20 \\
Total for Warp cell & 255 & 100 \\
\hline
\end{tabular}

Table 1: Implementation metrics for Warp cell

The IU handles data input/output between the host and the Warp array. The host-IU interface is streamlined by implementing a 32-bit wide interface, even though the Warp array has only 16-bit wide internal data paths. This arrangement is preferred because data transfers between host and IU are slower than the transfers between IU and the array. Data transfers between host and IU can be controlled by interrupts; in this case, the IU behaves like a slave device. The IU can also convert packed 8-bit or 16-bit integers transferred from the host into 32-bit floatingpoint numbers for the Warp array, and vice versa.

The IU is controlled by a 96-bit wide programmable micro-engine, which is similar to the Warp cell controller in programmability. The IU has several control registers that are mapped into the host address space; the host can control the IU and hence the Warp array by setting these registers. The IU has a power consumption of $82 \mathrm{~W}$ (typical) and $123 \mathrm{~W}$ (maximum). Table 2 presents implementation metrics for the $\mathrm{IU}$.

\begin{tabular}{|l|c|c|}
\hline \multicolumn{1}{|c|}{ Block in IU } & Chip count & Area contribution (\%) \\
\hline Data-converter & 44 & 19 \\
Address generator & 45 & 19 \\
Clock and host interface & 101 & 31 \\
Micro-engine & 49 & 20 \\
Other & 25 & 11 \\
Total for IU & 264 & 100 \\
\hline
\end{tabular}

Table 2: Implementation metrics for IU 


\section{Host system}

The Warp host controls the Warp array and other peripherals, supports fast data transfer rates to and from the Warp array, and also runs application code that cannot easily be mapped on the array. An overview of the host is presented in Figure 6. The host is partitioned into a standard workstation (the master) and an external host. The workstation provides a UNIX programming environment to the user, and also controls the external host. The external host consists of two cluster processors, a subsystem called support processor, and some graphics devices $(\mathrm{I}, \mathrm{O})$.

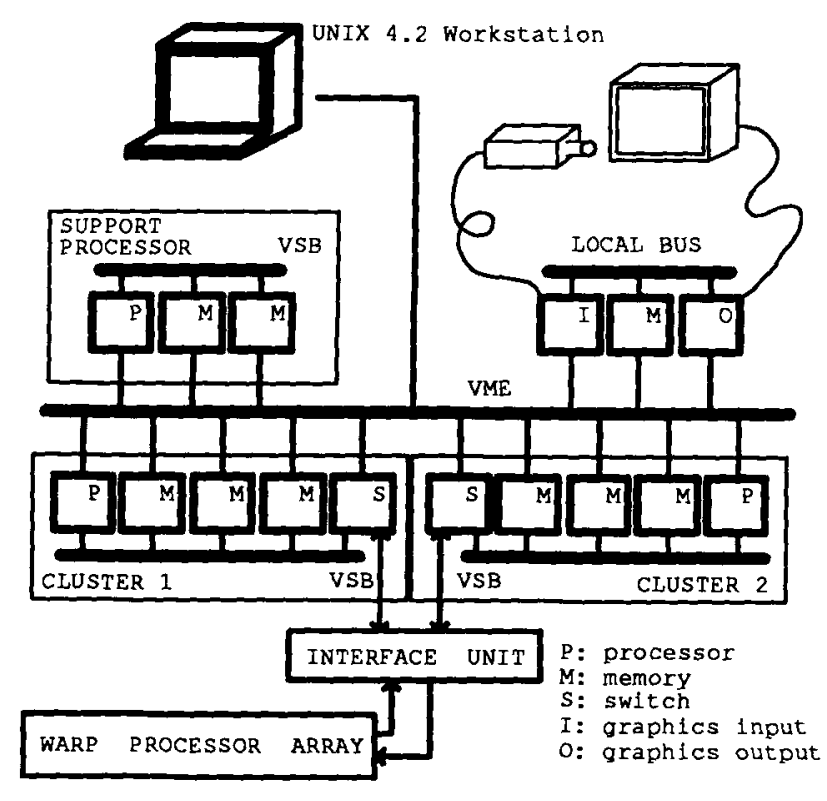

Figure 6: Host of the Warp machine

Control of the extemal host is strictly centralized: the workstation, the master processor, issues commands to the cluster and support processors through message buffers local to each of these processors. The two clusters work in parallel, each handling a unidirectional flow of data to or from the Warp processor, through the IU. The two clusters can exchange their roles in sending or receiving data for different phases of a computation, in a ping-pong fashion. An arbitration mechanism transparent to the user has been implemented to prohibit simultaneous writing or reading to the Warp array when the clusters switch roles. The support processor controls peripheral I/O devices and handles floating-point exceptions and other interrupt signals from the Warp array. These interrupts are serviced by the support processor, rather than by the master processor, to minimize interrupt response time. After servicing the interrupt, the support processor notifies the master processor.

The external host is built around a VME bus. The two clusters and the support processor each consist of a standalone MC68020 microprocessor (P) and a dual-ported memory (M), which can be accessed either via a local bus or via the global VME bus. The local bus is a VSB bus in the production machine and a VMX32 bus for the prototype; the major improvements of VSB over VMX32 are better support for arbitration and the addition of DMA-type accesses. Each cluster has a switch board $(S)$ for sending and receiving data to and from the Warp array, through the IU. The switch also has a VME interface, used by the master processor to start, stop, and control the Warp array. The VME bus of the master processor inside the workstation is connected to the VME bus of the external host via a bus-coupler (bus repeater). While the prototype Warp used a commercial bus-coupler, the PC Warp employs a custom-designed device. The difference between the two is that the custom-designed bus repeater decouples the external host VME bus from the Sun-3 VME bus: intra-bus transfers can occur concurrently on both busses. 
There are three memory banks inside each cluster processor to support concurrent memory accesses. For example, the first memory bank may be receiving a new set of data from an $1 / O$ device, while data in the second bank is transferred to the Warp array, and the third contains the cluster program code.

Presently, the memory of the external host are built out of 1-Mbyte memory boards; including the 3 Mbytes of memory on the processor boards, the total memory capacity of the external host is 11 Mbytes. An expansion of up to 59 Mbytes is possible by populating all the 14 available slots of the VME card cage with 4-Mbyte memory boards. Large data structures can be stored in these memories where they will not be swapped out by the operating system. This is important for consistent performance in real-time applications. The external host can also support special devices such as frame buffers and high speed disks. This allows the programmer to transfer data directly between Warp and other devices.

Except for the switch, all boards in the external host are off-the-shelf components. The industry standard boards allow us to take advantage of commercial processors, I/O boards, memory, and software. They also make the host an open system to which it is relatively easy to add new devices and interfaces to other computers. Moreover, standard boards provide a growth path for future system improvements with a minimal investment of time and resources. During the transition from prototype to production machine, faster processor boards (from $12 \mathrm{MHz}$ to 16 $\mathrm{MHz}$ ) and larger memories have been introduced, and they have been incorporated into the host with little effort.

\subsection{Host I/O bandwidth}

The Warp array can input a 32-bit word and output a 32-bit word every $200 \mathrm{~ns}$. Correspondingly, to sustain this peak rate, each cluster must be able to read or write a 32-bit data item every $200 \mathrm{~ns}$. This peak I/O bandwidth requirement can be satisfied if the input and output data are 8-bit or 16-bit integers that can be accessed sequentially.

In signal, image and low-level vision processing, the input and output data are usually 16- or 8-bit integers. The data can be packed into 32-bit words before being transferred to the $\mathrm{IU}$, which unpacks the data into two or four 32-bit floating-point numbers before sending them to the Warp array. The reverse operation takes place with the floating-point outputs of the Warp array. With this packing and unpacking, the data bandwidth requirement between the host and $\mathrm{IU}$ is reduced by a factor of two or four. Image data can be packed on the digitizer boards, without incurring overhead on the host. The commercial digitizer boards pack only two 8-bit pixels at a time; a frame-buffer capable of packing 4 pixels into a 32-bit word has been developed at Carnegie Mellon.

The I/O bandwidth of the PC Warp external host is greatly improved over that of the prototype machine [5]. The PC Warp supports DMA and uses faster processor and memory boards. If the data transfer is sequential, DMA can be used to achieve the transfer time of less than $500 \mathrm{~ns}$ per word. With block transfer mode, this transfer time is further reduced to about $350 \mathrm{~ns}$. The speed for non-sequential data transfers depends on the complexity of the address computation. For simple address patterns, one 32-bit word is transferred in about $900 \mathrm{~ns}$.

There are two classes of applications: those whose input/output data are pixel values (e.g., vision), and those whose input/output data are floating-point quantities (e.g., scientific computing). In vision applications, data are often transferred in raster order. By packing/unpacking the pixels and using DMA, the host $1 / O$ bandwidth can sustain the maximum bandwidth of all such programs. Many of the applications that need floating-point input and output data have non-sequential data access patterns. The host becomes a bottleneck if the rate of data transfer (and address generation if DMA cannot used) is lower than the rate the data is processed on the array. Fortunately, for many scientific applications, the computation per data item is typically quite large and the host $1 / O$ bandwidth is seldom the limiting factor in the performance of the array. 


\subsection{Host software}

The Warp host has a run-time software library that allows the programmer to synchronize the support processor and two clusters and to allocate memory in the external host. The run-time software also handles the communication and interrupts between the master and the processors in the external host. The library of run-time routines includes utilities such as copying and moving data within the host system, subwindow selection of images, and peripheral device drivers. The compiler generates program-specific input and output routines for the clusters so that a user needs not be concerned with programming at this level; these routines are linked at load time to the two cluster processor libraries.

The application program usually runs on the Warp array under control of the master; however, it is possible to assign sub-tasks to any of the processors in the external host. This decreases the execution time for two reasons: there is more parallelism in the computation, and data transfers between the cluster and the array using the VSB bus are twice as fast as transfers between the master processor and the array through the VME bus repeater. The processors in the external host have been extensively used in various applications, for example, obstacle avoidance for a robot vehicle and singular value decomposition.

Memory allocation and processor synchronization inside the external host are handled by the application program through subroutine calls to the run-time software. Memory is allocated through the equivalent of a UNIX malloc() system call, the only difference being that the memory bank has to be explicitly specified. This explicit control allows the user to fully exploit the parallelism of the system; for example, different processors can be programmed to access different memory banks through different busses concurrently.

Tasks are scheduled by the master processor. The application code can schedule a task to be run on the completion of a different task. Once the master processor determines that one task has completed, it schedules another task requested by the application code. Overhead for this run-time scheduling of tasks is minimal.

\section{Programming Warp}

As mentioned in the introduction, Warp is programmed in a language called W2. Programs written in W2 are translated by an optimizing compiler into object code for the Warp machine. W2 hides the low level details of the machine and allows the user to concentrate on the problem of mapping an application onto a processor array. In this section, we first describe the language and then some common computation partitioning techniques.

\subsection{The W2 language}

The W2 language provides an abstract programming model of the machine that allows the user to focus on parallelism at the array level. The user views the Warp system as a linear array of identical, conventional processors that can communicate asynchronously with their left and right neighbors. The semantics of the communication primitives are that a cell will block if it tries to receive from an empty queue or send to a full one. These semantics are enforced at compile time in the prototype and at run time in the PC Warp, as explained in Section 4.1.2.

The user supplies the code to be executed on each cell, and the compiler handles the details of code generation and scheduling. This arrangement gives the user full control over computation partitioning and algorithm design. The language for describing the cell code is Algol-like, with iterative and conditional statements. In addition, the language provides receive and send primitives for specifying intercell communication. The compiler handles the parallelism both at the system and cell levels. At the system level, the external host and the IU are hidden from the user. The compiler generates code for the host and the IU to transfer data between the host and the array. Moreover, for the prototype Warp, addresses and loop control signals are automatically extracted from the cell programs; they are generated on the IU and passed down the address queue. At the cell level, the pipelining and 
parallelism in the data path of the cells are hidden from the user. The compiler translates the Algol level constructs in the cell programs directly into horizontal microcode for the cells.

Figure 7 is an example of a $10 \times 10$ matrix multiplication program. Each cell computes one column of the result. We first load each cell with a column of the second matrix operand, then we stream the first matrix in row by row. As each row passes through the array, we accumulate the result for a column in each cell, and send the entire row of results to the host. The loading and unloading of data are slightly complicated because all cells execute the same program. Send and receive transfer data between adjacent cells; the first parameter determines the direction, and the second parameter selects the hardware channel to be used. The third parameter specifies the source (send) or the sink (receive). The fourth parameter, only applicable to those channels communicating with the host, binds the array input and output to the formal parameters of the cell programs. This information is used by the compiler to generate code for the host.

\subsection{Problem partitioning}

As discussed in Section 3, the architecture of the Warp array can support various kinds of algorithms: fine-grain or large-grain parallelism, local or global operations, homogeneous or heterogeneous. There are three general problem partitioning methods $[4,22]$ : input partitioning, output partitioning, and pipelining.

\subsubsection{Input partitioning}

In this model, the input data are partitioned among the Warp cells. Each cell computes on its portion of the input data to produce a corresponding portion of the output data. This model is useful in image processing where the result at each point of the output image depends only on a small neighborhood of the corresponding point of the input image.

Input partitioning is a simple and powerful method for exploiting parallelism-most parallel machines support it in one form or another. Many of the algorithms on Warp make use of it, including most of the low-level vision programs, the discrete cosine transform (DCT), singular value decomposition [2], connected component labeling [22], border following, and the convex hull. The last three algorithms mentioned also transmit information in other ways; for example, connected components labeling first partitions the image by rows among the cells, labels each cell's portion separately, and then combines the labels from different portions to create a global labeling.

\subsubsection{Output partitioning}

In this model, each Warp cell processes the entire input data set or a large part of it, but produces only part of the output. This model is used when the input to output mapping is not regular, or when any input can influence any output. Histogram and image warping are examples of such computations. This model usually requires a lot of memory because either the required input data set must be stored and then processed later, or the output must be stored in memory while the input is processed, and then output later. Each Warp cell has $32 \mathrm{~K}$ words of local memory to support efficient use of this model.

\subsubsection{Pipelining}

In this model, typical of systolic computation, the algorithm is partitioned among the cells in the array, and each cell performs one stage of the processing. The Warp array's high inter-cell communication bandwidth and effectiveness in handling fine-grain parallelism make it possible to use this model. For some algorithms, this is the only method of achieving parallelism that is possible.

A simple example of the use of pipelining is the solution of elliptic partial differential equations using successive over-relaxation [36]. Consider the following equation: 


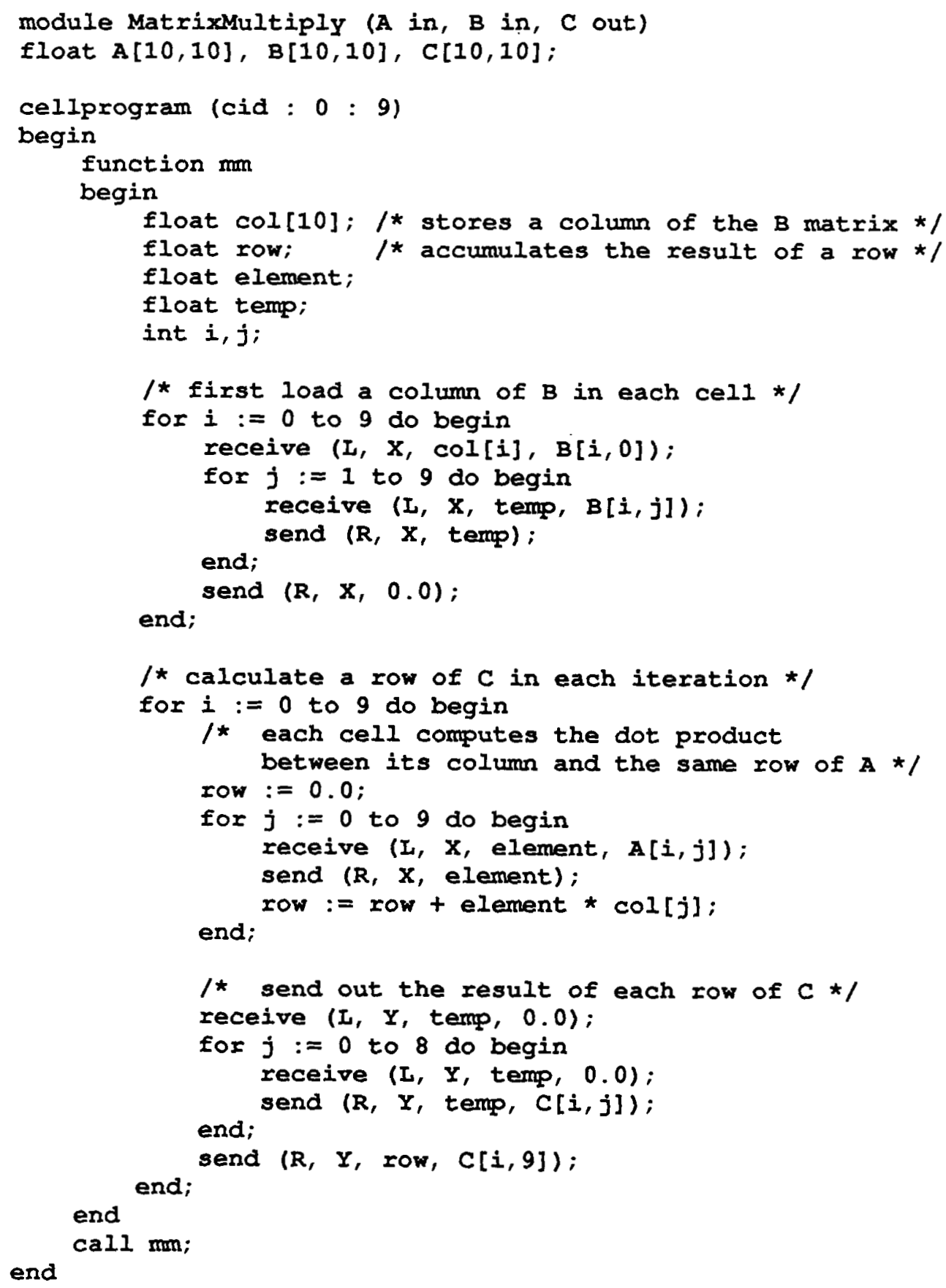

Figure 7: Example program

$$
\frac{\partial^{2} u}{\partial x^{2}}+\frac{\partial^{2} u}{\partial y^{2}}=f(x, y) .
$$

The system is solved by repeatedly combining the current values of $u$ on a 2-dimensional grid using the following recurrence:

$$
u_{i, j}^{\prime}=(1-\omega) u_{i, j}+\omega \frac{f_{i, j}+u_{i, j-1}+u_{i, j+1}+u_{i+1, j}+u_{i-1, j}}{4}, \quad \text { where } \omega \text { is a constant parameter. }
$$

In the Warp implementation, each cell is responsible for one relaxation, as expressed by the above equation. In 
raster order, each cell receives inputs from the preceding cell, performs its relaxation step, and outputs the results to the next cell. While a cell is performing the $k^{\text {th }}$ relaxation step on row $i$, the preceding and next cells perform the $k-1^{\text {st }}$ and $k+1^{\text {st }}$ relaxation steps on rows $i+2$ and $i-2$, respectively. Thus, in one pass of the $u$ values through the 10-cell Warp array, the above recurrence is applied ten times. This process is repeated, under control of the external host, until convergence is achieved.

\section{Evaluation}

Since the two copies of the wire-wrapped prototype Warp machine became operational at Carnegie Mellon in 1986 , we have used the machines substantially in various applications $[2,3,4,10,13,22]$. The application effort has been increased since April 1987 when the first PC Warp machine was delivered to Carnegie Mellon.

The applications area that guided the development of Warp most strongly was computer vision, particularly as applied to robot navigation. We studied a standard library of image processing algorithms [30] and concluded that the great majority of algorithms could efficiently use the Warp machine. Moreover, robot navigation is an area of active research at Carnegie Mellon and has real-time requirements where Warp can make a significant difference in overall performance $[32,33]$. Since the requirements of computer vision had a significant influence on all aspects of the design of Warp, we contrast the Warp machine with other architectures directed towards computer vision in Section 8.2.

Our first effort was to develop applications that used Warp for robot navigation. Presently mounted inside of a robot vehicle for direct use in vehicle control, Warp has been used to perform road following and obstacle avoidance. We have implemented road following using color classification, obstacle avoidance using stereo vision, obstacle avoidance using a laser range-finder, and path planning using dynamic programming. We have also implemented a significant portion (approximately 100 programs) of an image processing library on Warp [30], to support robot navigation and vision research in general. Some of the library routines are listed in Table 4.

A second interest was in using Warp in signal processing and scientific computing. Warp's high floating-point computation rate and systolic structure make it especially attractive for these applications. We have implemented singular value decomposition (SVD) for adaptive beamforming, fast two-dimensional image correlation using FFT, successive over-relaxation (SOR) for the solution of elliptic partial differential equations (PDE), as well as computational geometry algorithms such as convex hull and algorithms for finding the shortest paths in a graph.

\subsection{Performance data}

Two figures of merit are used to evaluate the performance of Warp. One is overall system performance, and the other is performance on specific algorithms. Table 3 presents Warp's performance in several systems for robot navigation, signal processing, scientific computation, and geometric algorithms, while Table 4 presents Warp's performance on a large number of specific algorithms. Both tables report the performance for the wire-wrapped Warp prototype with a Sun-3/160 as the master processor. The PC Warp will in general exceed the reported performance, because of its improved architecture and increased host $\mathrm{I} / \mathrm{O}$ speed as described earlier. Table 3 includes all system overheads except for initial program memory loading. We compare Warp performance with a Vax $11 / 780$ with floating-point accelerator, because this computer is widely used and therefore familiar to most people.

Statistics have been gathered for a collection of $72 \mathrm{~W} 2$ programs in the application areas of vision, signal processing and scientific computing [23]. Table 4 presents the utilization of the Warp array for a sample of these programs. System overheads such as microcode loading and program initialization are not counted. We assume that the host $\mathrm{Y} / \mathrm{O}$ can keep up with the Warp array; this assumption is realistic for most applications with the host of the 
production Warp machine. Figure 8 shows the performance distribution of the 72 programs. The arithmetic mean is 28 MFLOPS, and the standard deviation is 18 MFLOPS.

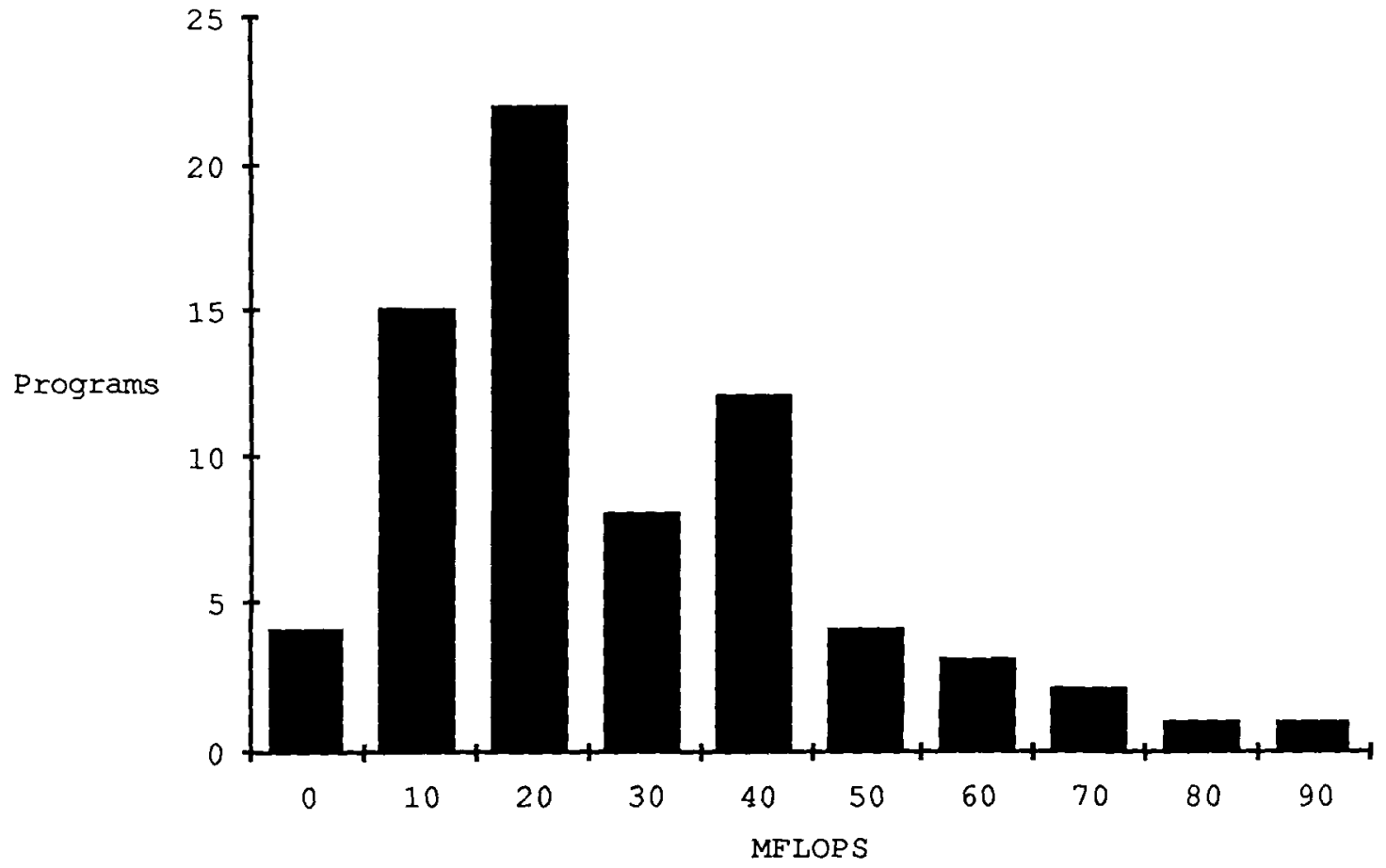

Figure 8: Performance distribution of a set of $72 \mathrm{~W} 2$ programs

The Warp cell has several independent functional units, including separate floating-point units for addition and multiplication. The achievable performance of a program is limited by the most used resource. For example, in a computation that contains only additions and no multiplications, the maximum achievable performance is only 50 MFLOPS. Table 4 gives an upper bound on the achievable performance and the achieved performance. The upper bound is obtained by assuming that the floating-point unit that is used more often in the program is the most used resource, and that it can be kept busy all the time. That is, this upper bound cannot be met even with a perfect compiler if the most used resource is some other functional unit, such as the memory, or if data dependencies in the computation prevent the most used resource from being used all the time.

Many of the programs in Tables 3 and 4 are coded without fine tuning the W2 code. Optimizations can often provide a significant speedup over the times given. First, the W2 code can be optimized, using conventional programming techniques such as unrolling loops with few iterations, replacing array references by scalars, and so on. Second, in some cases in Table 3 the external host in the prototype Warp is a bottleneck, and it is possible to speed up this portion of the Warp machine by recoding the I/O transfer programs generated by the W2 compiler into MC68020 assembly language. Moreover, the external host for the PC Warp is faster and supports DMA, so that even with the compiler generated code it will no longer be the bottleneck. Third, since restrictions on using the Warp cells in a pipeline are removed in PC Warp as explained in Section 4.3.4, it will be possible to implement many of the vision algorithms in a pipelining fashion. This can lead to a three-fold speedup, since input, computation, and output will be done at the same time. Fourth, in a few cases we have discovered a better algorithm for the Warp implementation than what was originally programmed.

In Table 3, the speedup ranges from 60 to 500. With the optimizations we discuss above, all systems listed should show at least a speedup of about 100 over the Vax $11 / 780$ with floating-point accelerator. 
Task

Time (ms)

Speedup over Vax 11/780

(All images are $512 \times 512$. All code compiler generated.) with floating-point accelerator

Quadratic image warping

400

100

Warp array generates addresses using quadratic form in $240 \mathrm{~ms}$.

Host computes output image using addresses generated by Warp.

Road-following

6000

200

Obstacle avoidance using ERIM, a laser range-finder

350

60

Time does not include $500 \mathrm{~ms}$ for scanner $\mathrm{I} / \mathrm{O}$.

Minimum-cost path, $512 \times 512$ image, one pass

500

60

Host provides feedback.

Detecting lines by Hough Transform

2000

387

Host merges results.

Minimum-cost path, 350-node graph

16000

98

Convex hull, 1,000 random nodes

Solving elliptic PDE by SOR, 50,625 unknowns (10 iterations)

18

74

Singular value decomposition of $100 \times 100$ matrix

180

440

FFT on 2D image

1500

100

Warp array takes $600 \mathrm{~ms}$. Remaining time is for data shuffling by host.

Image correlation using FFT

7000

300

Data shuffling in host.

Image compression with $8 \times 8$ discrete cosine transforms

110

500

Mandelbrot image, 256 iterations

6960

100

Table 3: Measured speedups on the wire-wrapped prototype Warp machine 


\subsection{Architectural Alternatives}

We discuss the architectural decisions made in Warp by contrasting them with the decisions made in bit-serial processor arrays, such as the Connection Machine [34] and MPP [7]. We chose these architectures because they have also been used extensively for computer vision and image processing, and because the design choices in these architectures were made significantly differently than in Warp. These differences help exhibit and clarify the design space for the Warp architecture.

We attempt to make our comparison quantitative by using benchmark data from a DARPA Image Understanding ("DARPA IU") workshop held in November 1986 to compare various computers for vision [29]. In this workshop, benchmarks for low and mid-level computer vision were defined and programmed by researchers on a wide variety of computers, including Warp and the Connection Machine [25].

We briefly review salient features of the Connection Machine, called CM-1, used in these benchmarks. It is a SIMD machine, consisting of an array of $64 \mathrm{~K}$ bit-serial processing elements, each with $4 \mathrm{~K}$ bits of memory. The processors are connected by two networks: one connects each processor to four adjacent processors, and the other is a 12-dimensional hypercube, connecting groups of 16 processors. The array is controlled by a host, which is a Symbolics 3640 Lisp machine. CM-1 is programmed in an extension to Common Lisp called *Lisp [24], in which references to data objects stored in the CM-1 array and objects on the host can be intermixed.

Although our intention is to illustrate architectural decisions made in Warp, not to compare it with the Connection Machine, we should not cite benchmark performance figures on two different computers without mentioning two critical factors, namely cost and size. CM-1 is approximately one order of magnitude more expensive and larger than Warp.

\subsubsection{Programming model}

Bit-serial processor arrays implement a data parallel programming model, in which different processors process different elements of the data set. In the Connection Machine, the programmer manipulates data objects stored in the Connection Machine array by the use of primitives in which the effect of a Lisp operator is distributed over a data object.

In systolic arrays, the systolic processors individually manipulate words of data. In Warp, we have implemented data parallel programming models through the use of input and output partitioning. We have encapsulated input partitioning over images in a specialized language called Apply [14]. In addition to these models, the high interprocessor bandwidth of the systolic array allows efficient implementation of pipelining, in which not the data, but the algorithm is partitioned.

\subsubsection{Processor I/O bandwidth and topology}

Systolic arrays have high bandwidth between processors, which are organized in a simple topology. In the case of the Warp array, this is the simplest possible topology, namely a linear array. The interconnection networks in the Connection Machine allow flexible topology, but low bandwidth between communicating processors.

Bit-serial processing arrays may suffer from a serious bottleneck in $\mathrm{I} / \mathrm{O}$ with the external world, because of the difficulty of feeding a large amount of data through a single simple processor. This bottleneck has been addressed in various ways. MPP uses a "staging memory" in which image data can be placed and distributed to the array along one dimension. The $I / O$ bottleneck has been addressed by a new version of the Connection Machine, called CM-2 [31]. In this computer, a number of disk drives can feed data into various points in the array simultaneously. The CM-1 benchmark figures do not include image I/O: the processing is done on an image which has already been loaded into the array, and processing is completed with the image still in the array. Otherwise, image I/O would 


\begin{tabular}{|c|c|c|c|}
\hline $\begin{array}{l}\text { Task } \\
\text { All images are } 512 \times 512 . \quad \text { All code compiler generated. }\end{array}$ & Time (ms) & $\begin{array}{l}\text { MFLOPS } \\
\text { (Upper bound) }\end{array}$ & $\begin{array}{l}\text { MFLOPS } \\
\text { (Achieved) }\end{array}$ \\
\hline $100 \times 100$ matrix multiplication. & 25 & 100 & 79 \\
\hline $3 \times 3$ convolution. & 70 & 94 & 66 \\
\hline $11 \times 11$ symmetric convolution. & 367 & 90 & 59 \\
\hline Calculate transformation table for non-linear warping. & 248 & 80 & 57 \\
\hline $\begin{array}{l}\text { Generate matrices for plane fit } \\
\text { for obstacle avoidance using ERIM scanner. }\end{array}$ & 174 & 62 & 49 \\
\hline Generate mapping table for affine image warping. & 225 & 67 & 43 \\
\hline Moravec's interest operator. & 82 & 60 & 36 \\
\hline $3 \times 3$ maximum filtering. & 280 & 67 & 30 \\
\hline Sobel edge detection. & 206 & 77 & 30 \\
\hline Label color image using quadratic form for road following. & 308 & 87 & 27 \\
\hline Image magnification using cubic spline interpolation. & 8438 & 66 & 25 \\
\hline $7 \times 7$ average gray values in square neighborhood. & 1090 & 51 & 24 \\
\hline $5 \times 5$ convolution. & 284 & 52 & 23 \\
\hline Calculate quadratic form from labelled color image. & 134 & 58 & 22 \\
\hline Compute gradient using $9 \times 9$ Canny operator. & 473 & 92 & 21 \\
\hline Discrete cosine transform on $8 \times 8$ windows. & 175 & 94 & 21 \\
\hline $3 \times 3$ Laplacian edge detection. & 228 & 94 & 20 \\
\hline 15×15 Harwood-style symmetric edge preserving smoothing. & 32000 & 50 & 16 \\
\hline Find zero-crossings. & 179 & 78 & 16 \\
\hline Calculate $(x, y, z)$ coordinates from ERIM laser range scanner data. & 24 & 75 & 13 \\
\hline Histogram. & 67 & 50 & 12 \\
\hline Coarse-to-fine correlation for stereo vision. & 12 & 77 & 11 \\
\hline $3 \times 3$ median filter. & 448 & 50 & 7 \\
\hline Levialdi's binary shrink operation. & 180 & 71 & 7 \\
\hline $31 \times 31$ average gray values in square neighborhood. & 444 & 61 & 5 \\
\hline Convert real image to integer using max, min linear scaling. & 249 & 66 & 4 \\
\hline Average $512 \times 512$ image to produce $256 \times 256$ & 150 & 58 & 3 \\
\hline
\end{tabular}

Table 4: Performance of specific algorithms on the wire-wrapped prototype Warp machine 
completely dominate processing time. In many cases it is necessary to process an image which is stored in a frame buffer or host memory, which is easier in Warp because of the high bandwidth between the Warp array and the Warp host. All the Warp benchmarks in this section include I/O time from the host.

The high bandwidth connection between processors in the Warp array makes it possible for all processors to see all data in an image, while achieving useful image processing time. (In fact, because of the linear topology, there is no time advantage to limit the passage of an image through less than all processors). This is important in global image computations such as Hough transform, where any input can influence any output. For example, the transform of a $512 \times 512$ image into a $180 \times 512$ Hough space took 1.7 seconds on Warp, only 2.5 times as long as on CM-1. The ratio here is far less than for a simple local computation on a large image, such as Laplacian and zero crossing.

In some global operations, processing is done separately on different cells, then combined in a series of pairwise merge operations using a "divide and conquer" approach. This type of computation can be difficult to implement using limited topology communications as in Warp. For example, in the Warp border following algorithm for a $512 \times 512$ image, individual cells trace the borders of different portions of the image, then those borders are combined in a series of merge operations in the Warp array. The time for border following on Warp is 1100 milliseconds, significantly more than the 100 milliseconds the algorithm takes on CM-1.

\subsubsection{Processor number and power}

Warp has only ten parallel processing elements in its array, each of which is a powerful 10 MFLOPS processor. $\mathrm{CM}-1$, on the other hand, has $64 \mathrm{~K}$ processing elements, each of which is a simple bit-serial processor. Thus, the two machines stand at opposite ends of the spectrum of processor number and power.

We find that the small number of processing elements in Warp makes it easier to get good use of the Warp array in problems where a complex global computation is performed on a moderate sized dataset. In these problems, not much data parallelism is "available." For example, the DARPA IU benchmarks included the computation of the two-dimensional convex hull [26] of a set of 1000 points. The CM-1 algorithm used a brush-fire expansion algorithm, which led to an execution time of 200 milliseconds for the complete computation. The same algorithm was implemented on Warp, and gave the 18 millisecond figure reported in Table 3. Similar ratios are found in the times for the minimal spanning tree of 1000 points (160 milliseconds on Warp versus 2.2 seconds on CM-1) and a triangle visibility problem for 1000 three dimensional triangles (400 milliseconds on Warp versus 1 second on CM-1).

Simple algorithms at the lowest level of vision, such as edge detection computations, run much faster on large arrays of processors such as the Connection Machine than Warp. This is because no communication is required between distant elements of the array, and the large array of processors can be readily mapped onto the large image array. For example, the computation of an $11 \times 11$ Laplacian [15] on a $512 \times 512$ image, followed by the detection of zero crossings, takes only 3 milliseconds on CM-1, as opposed to 400 milliseconds on Warp.

The floating-point processors in Warp aid the programmer in eliminating the need for low-level algorithmic analysis. For example, the Connection Machine used discrete fixed point approximation to several algorithms, including Voronoi diagram and convex hull. The use of floating-point made it unnecessary for the Warp programmer to make assumptions about the data range and distribution.

In conclusion, we see that bit-serial processor arrays excel in the lowest level of vision, such as edge detection. The CM-1's performance at this level exceeded Warp's by two orders of magnitude. However, specialized hardware must be used to eliminate a severe I/O bottleneck to actually observe this performance. The use of the 
router in the Connection Machine allows it to do well also at higher levels of vision, such as border following. We also see that the more general class of programming models and use of floating-point hardware in Warp give it good actual performance in a wide range of algorithms, especially including complex global computations on moderate sized data sets.

\section{Conclusions}

The Warp computer has achieved high performance in a variety of application areas, including low-level vision, signal processing and scientific computation. Currently produced by our industrial partner (GE), Warp is much more powerful and programmable than many other machines of comparable cost.

The effectiveness of the Warp computer results from a balanced effort in architecture, software and applications. The simple, linear topology of the Warp array naturally supports several useful problem partitioning models; the Warp cells' high degree of programmability and large local memory make up for the lack of higher dimensional connectivity. The high computation rate on each cell is matched by an equally high inter-and intra-cell bandwidth. The host system provides the Warp array with high I/O bandwidth. The optimizing W2 compiler maps programs from a high-level language to efficient microcode for the Warp array. Integration of the Warp array into UNIX as an attached processor makes the Warp machine easily accessible to users. A sizable application library has been implemented to support development of research systems in vision.

The development of a compiler is essential in designing the architecture of a machine. Designing and implementing a compiler require a thorough study of the functionality of the machine; the systematic analysis of the machine allows us to uncover problems that may otherwise be undetected by writing sample programs. The compiler is also an excellent tool for evaluating different architectural alternatives. The development of the W2 compiler has significantly influenced the evolution of the architecture of Warp.

An early identification of an application area is essential for the development of an experimental machine such as Warp whose architecture is radically different from conventional ones. Including the application users in the early phase of the project - the vision research group at Carnegie Mellon in our case-helped us focus on the architectural requirements and provided early feedback.

Prototyping is important for architecture development. An early prototype system gives the designers realistic feedback about the constraints of the hardware implementation and provides a base for the software and application developers to test out their ideas. To speed up implementation of the prototype, we used off-the-shelf parts. To concentrate our efforts on the architecture of the Warp array, we developed the host from industry standard boards.

The Warp machine has demonstrated the feasibility of programmable, high-performance systolic array computers. The programmability of Warp has substantially extended the machine's application domain. The cost of programmability is limited to an increase in the physical size of the machine; it does not incur a loss in performance, given appropriate architectural support. This is shown by Warp, as it can be programmed to execute many well-known systolic algorithms as fast as special-purpose arrays built using similar technology.

\section{Acknowledgments}

We appreciate the contributions to the Warp project by our colleagues and visitors at Carnegie Mellon: D. Adams, F. Bitz, C. Bono, M. Browne, B. Bruegge, C. H. Chang, E. Clune, R. Cohn, R. Conde, J. Deutch, P. Dew, B. Enderton, L. Hamey, P. K. Hsiung, K. Hughes, T. Kanade, G. Klinker, P. Lieu, P. Maulik, D. Morris, A. Noaman, T. M. Parng, H. Printz, J. Race, M. Ravishankar, J. Rendas, H. Ribas, C. Sarocky, K. Sarocky, J. Senko, 
Y. Shintani, B. Siegell, H. Sohn, P. Steenkiste, Y. B. Tsai, P. S. Tseng, R. Wallace, J. K. Wang, I. C. Wu, D. Yam, and A. Zobel. We thank our industrial partners GE and Honeywell for their contribution towards the construction of the wire-wrapped prototypes. We appreciate the continued collaboration with GE for the development of the production Warp machine. In particular, we thank R. Barned, S. Carmel, J. Cokus, J. Condon, D. Crump, R. A. Field, R. Gaus, N. Gearhart, J. Iannuzzi, A. Lock, C. Pickering, A. Pfueller, M. Sarig, S. Sillich, T. Stark, W. Tates, A. Toll, C. Walrath, and J. Weimar of GE in Syracuse for their efforts.

\section{References}

1. Annaratone, M., Arnould, E., Gross, T., Kung, H. T., Lam, M. S., Menzilcioglu, O., Sarocky, K., and Webb, J. A. Warp Architecture and Implementation. Proceedings of the 13th Annual International Symposium on Computer Architecture, IEEE/ACM, June, 1986, pp. 346 - 356.

2. Annaratone, M., E. Arnould, H.T. Kung, and O. Menzilcioglu. Using Warp as a Supercomputer in Signal Processing. Proceedings of ICASSP 86, April, 1986, pp. 2895-2898.

3. Annaratone, M., Bitz, F., Clune E., Kung H. T., Maulik, P., Ribas, H., Tseng, P., and Webb, J. Applications and Algorithm Partitioning on Warp. Proc. Compcon Spring 87, San Francisco, February, 1987, pp. 272-275.

4. Annaratone, M., Bitz, F., Deutch, J., Hamey, L., Kung, H. T., Maulik P. C., Tseng, P., and Webb, J. A. Applications Experience on Warp. Proceedings of the 1987 National Computer Conference, AFIPS, Chicago, June, 1987, pp. 149-158.

5. Annaratone, M., Arnould, E., Cohn, R., Gross, T., Kung, H. T., Lam, M., Menzilcioglu, O., Sarocky, K., Senko, J., and Webb, J. Architecture of Warp. Proc. Compcon Spring 87, San Francisco, February, 1987, pp. 264-267.

6. Annaratone, M., Arnould, E., Cohn, R., Gross, T., Kung, H. T., Lam, M., Menzilcioglu, O., Sarocky, K., Senko, J., and Webb, J. Warp Architecture: From Prototype to Production. Proceedings of the 1987 National Computer Conference, AFIPS, Chicago, June, 1987, pp. 133-140.

7. Batcher, K. E. "Design of a Massively Parallel Processor". IEEE Trans. on Computers C-29 (1980), 836-840.

8. Bruegge, B., Chang C., Cohn R., Gross T., Lam M., Lieu P., Noaman A., and Yam, D. The Warp Programming Environment. Proceedings of the 1987 National Computer Conference, AFIPS, Chicago, June, 1987, pp. 141-148.

9. Charlesworth, A. E. "An Approach to Scientific Array Processing: The Architectural Design of the AP-120B/FPS-164 Family". Computer 14 (September 1981), 18-27.

10. Clune, E., Crisman, J. D., Klinker, G. J., and Webb, J. A. Implementation and Performance of a Complex Vision System on a Systolic Array Machine. Tech. Rept. CMU-RI-TR-87-16, Robotics Institute, Carnegie Mellon University, 1987.

11. Fisher, A. L., Kung, H. T. and Sarocky, K. Experience with the CMU Programmable Systolic Chip. Microarchitecture of VLSI Computers, 1985, pp. 209 - 222.

12. Gross, T. and Lam, M. Compilation for a High-performance Systolic Array. Proceedings of the SIGPLAN 86 Symposium on Compiler Construction, ACM SIGPLAN, June, 1986, pp. 27-38.

13. Gross, T., Kung, H. T., Lam, M. and Webb, J. Warp as a Machine for Low-Level Vision. Proceedings of 1985 IEEE International Conference on Robotics and Automation, March, 1985, pp. 790-800.

14. Hamey, L. G. C., Webb, J. A., and Wu, I. C. Low-level Vision on Warp and the Apply Programming Model. In Parallel Computation and Computers for Artificial Intelligence, Kluwer Academic Publishers, 1987. Edited by J. Kowalik.

15. Haralick, R. M. "Digital Step Edges from Zero Crossings of Second Directional Derivatives". IEEE Transactions on Pattern Analysis and Machine Intelligence 6 (1984), 58-68.

16. Hsu, F.H., Kung, H.T., Nishizawa, T. and Sussman, A. Architecture of the Link and Interconnection Chip. Proceedings of 1985 Chapel Hill Conference on VLSI, Computer Science Department, The University of North Carolina, May, 1985, pp. 186-195. 
17. Kanade, T., and Webb, J. A. End of Year Report for Parallel Vision Algorithm Design and Implementation. Robotics Institute, Carnegie Mellon University, 1987.

18. Kung, H.T. "Why Systolic Architectures?". Computer Magazine 15, 1 (Jan. 1982), 37-46.

19. Kung, H.T. Systolic Algorithms for the CMU Warp Processor. Proceedings of the Seventh International Conference on Pattern Recognition, International Association for Pattern Recognition, 1984, pp. 570-577.

20. Kung, H. T. "Memory Requirements for Balanced Computer Architectures". Journal of Complexity 1, 1 (1985), 147-157.

21. Kung, H.T. and Webb, J.A. Global Operations on the CMU Warp Machine. Proceedings of 1985 AIAA Computers in Aerospace V Conference, American Institute of Aeronautics and Astronautics, October, 1985 , pp. 209-218.

22. Kung, H. T. and Webb, J. A. "Mapping Image Processing Operations onto a Linear Systolic Machine". Distributed Computing 1, 4 (1986), 246-257.

23. Lam, M. S. A Systolic Array Optimizing Compiler. Ph.D. Th., Carnegie Mellon University, May 1987.

24. Lasser, C. The Complete *Lisp Manual. Thinking Machines Corporation, Cambridge, Massachusetts, 1986.

25. Little, J. J., Glelloch, G., and Cass, T. Parallel Algorithms for Computer Vision on the Connection Machine. Image Understanding Workshop, DARPA, Feb, 1987, pp. 628-638.

26. Preparata, F. P. and Shamos, M. I.. Computational Geometry - An Introduction. Springer, New York, 1985.

27. Rau, B. R. and Glaeser, C. D. . Some Scheduling Techniques and an Easily Schedulable Horizontal Architecture for High Performance Scientific Computing. Proc. 14th Annual Workshop on Microprogramming, October, 1981, pp. 183-198.

28. Rau, B. R., Kuekes, P. J. and Glaeser, C. D. A Statically Scheduled VLSI Interconnect for Parallel Processors. VLSI Systems and Computations, October, 1981, pp. 389-395.

29. Rosenfeld, A. A Report on the DARPA Image Understanding Architectures Workshop. Image Understanding Workshop, DARPA, Los Angeles, California, February, 1987, pp. 298-301.

30. Tamura, H., Sakane, S., Tomita, F., Yokoya, N., Sakaue, K. and Kaneko, N. SPIDER Users' Manual. Joint System Development Corp., Tokyo, 1983.

31. Thinking Machines Corporation. Connection Machine Model CM-2 Technical Summary. HA 87-4, Thinking Machines Corporation, April, 1897.

32. Wallace, R., Stentz, A., Thorpe, C., Whittaker, W. and Kanade, T. First Results in Robot Road-Following. Proceedings of IJCAI, 1985, pp. 1089-1093.

33. Wallace, R., Matsuzaki, K., Goto, Y., Crisman, J., Webb, J. and Kanade, T. Progress in Robot Road-Following. Proceedings of 1986 IEEE International Conference on Robotics and Automation, April, 1986, pp. 1615-1621.

34. Waltz, D. L. "Applications of the Connection Machine". IEEE Computer 20, 1 (January 1987), 85-97.

35. Woo, B., Lin, L. and Ware, F. A High-Speed 32 Bit IEEE Floating-Point Chip Set for Digital Signal Processing. Proceedings of ICASSP 84, IEEE, 1984, pp. 16.6.1-16.6.4.

36. Young, D.. Iterative Solution of Large Linear Systems. Academic Press, New York, 1971. 
